\title{
Stretching the spectra of Kerr frequency combs with self-adaptive boundary silicon waveguides
}

\author{
Jianhao Zhang, ${ }^{a, *}$ Vincent Pelgrin, ${ }^{a}$ Carlos Alonso-Ramos, ${ }^{a}$ Laurent Vivien, ${ }^{a}$ Sailing He, ${ }^{b}$ and Eric Cassan ${ }^{a, *}$ \\ aUniversité Paris-Saclay, CNRS, Centre de Nanosciences et de Nanotechnologies, Palaiseau, France \\ ${ }^{\text {b}}$ Zhejiang University, Centre for Optical and Electromagnetic Research, State Key Laboratory for Modern Optical Instrumentation, Hangzhou, China
}

\begin{abstract}
Dispersion engineering of optical waveguides is among the most important steps in enabling the realization of Kerr optical frequency combs. A recurring problem is the limited bandwidth in which the nonlinear phase matching condition is satisfied, due to the dispersion of the waveguide. This limitation is particularly stringent in high-index-contrast technologies such as silicon-on-insulator. We propose a general approach to stretch the bandwidth of Kerr frequency combs based on subwavelength engineering of single-mode waveguides with self-adaptive boundaries. The wideband flattened dispersion operation comes from the particular property of the waveguide optical mode that automatically self-adapts its spatial profile at different wavelengths to slightly different effective spatial spans determined by its effective index values. This flattened dispersion relies on the squeezing of small normal-dispersion regions between two anomalous spectral zones, which enables it to achieve two Cherenkov radiation points and substantially broaden the comb, achieving a bandwidth between 2.2 and $3.4 \mu \mathrm{m}$ wavelength. This strategy opens up a design space for trimming the spectra of Kerr frequency combs using high-index-contrast platforms and can provide benefits to various nonlinear applications in which the manipulation of energy spacing and phase matching are pivotal.
\end{abstract}

Keywords: nonlinear optics; effective boundary; subwavelength grating; silicon frequency comb.

Received Feb. 23, 2020; revised manuscript received May 26, 2020; accepted for publication Jun. 1, 2020; published online Jun. 23, 2020.

(C) The Authors. Published by SPIE and CLP under a Creative Commons Attribution 4.0 Unported License. Distribution or reproduction of this work in whole or in part requires full attribution of the original publication, including its DOI.

[DOI: 10.1117/1.AP.2.4.046001]

\section{Introduction}

Third-order nonlinear optical processes in photonic waveguides have raised increasing interest in recent years, due to their unique capabilities for on-chip light generation and for the manipulation of guided light in spectral/temporal domains, with an immense potential for the implementation of light sources based on optical parametric amplification, supercontinuum, or frequency comb generation processes. ${ }^{1-9}$ Kerr frequency comb generation has great potential for applications in a wide range of fields, from metrology-on-a-chip to the synthesis of radiofrequency signals or integrated spectroscopy. ${ }^{10-19}$ Comprising discrete and equally spaced frequency lines, the Kerr frequency combs require precise control of the waveguide dispersion and nonlinearity, the gain and loss of optical waveguides, especially when temporal patterns with few solitons are expected. ${ }^{20}$

*Address all correspondence to Eric Cassan, E-mail: eric.cassan @ universite-parissaclay.fr; Jianhao Zhang, E-mail: jianhao.zhang@universite-paris-saclay.fr
Controlling the chromatic dispersion of optical waveguides is a key preliminary step for the exploitation of Kerr frequency combs. As such, the problem is quite simple in principle: it is essentially a question of optimizing the geometrical dimensions of the waveguides in order to compensate for the material natural dispersion as well as that provided by the nonlinear effect itself, in order to satisfy, as a whole, the energy and momentum conservation conditions. To balance the nonlinearity-induced wavevector phase mismatch, anomalous dispersion is generally envisaged, ${ }^{10,11,20}$ it can be supported directly from the materials (e.g., silica at telecom wavelengths) or induced by the waveguide dispersion with well-designed waveguide cross-sections. As a result, toroidal-shape cavities using silica ${ }^{10}$ or $\mathrm{MgF}_{2}{ }^{12,13}$ are frequently used for frequency comb generation due to their ultrahigh $Q$ factors of up to a few millions and to the low dispersion in these structures. Another classical material platform used for frequency comb demonstrations is based on silicon nitride ( $\mathrm{SiN}$ ) waveguides. Due to the high quality-factors of $\mathrm{SiN}$ microring resonators (up to $\sim 10^{6}$ ) and a nonlinear Kerr index 
around $2.4 \times 10^{-19} \mathrm{~m}^{2} / \mathrm{W}$, high-performance frequency combs extending two octaves can be achieved on-chip. ${ }^{15-18}$ Despite the merits and inherent advantages of this platform, the SiN waveguides are less attractive for compact photonic integration than silicon ones. The realization of Kerr frequency combs directly in $\mathrm{Si}$ waveguides thus presents great interest. However, achieving broadband phase-matching in $\mathrm{Si}$ waveguides is difficult due to the high-index contrast between $\mathrm{Si}$ and $\mathrm{SiO}_{2}(\Delta n \approx 2)$ that makes the dispersion engineering condition highly sensitive to wavelength. ${ }^{5,21}$ It appears that, by moving from silicon nitride to silicon waveguides, an advantage (a lower pump power) and a drawback (a reduced frequency comb spectral width) result simultaneously. Consequently, addressing the problem of the spectral bandwidth of microcombs in silicon waveguides is an important point. Broadband phase-matching was shown based on the optimization of high order dispersion terms, e.g., by relying on fourth-order dispersive waveguides, ${ }^{22-26}$ or by implementing slightly etched rib geometries. ${ }^{6}$ Yet, the proposed solutions require complex fabrication processes, with deposition of different materials or a tight control of the rib and slab thicknesses, respectively. Though mode-locked pulse generation has been achieved using normal-dispersion, ${ }^{27}$ a flexible method to flatten the silicon waveguide dispersion and trim the spectra for Kerr frequency comb generation is still expected. The feasibility of spectrum engineering ${ }^{28}$ could further enhance optical frequency combs as strong candidates for the realization of onchip silicon light sources ${ }^{29}$ and could provide a strong basis for many applications in on-chip spectroscopy or metrology, ${ }^{30}$ as well as for emerging research domains such as time-spacefrequency mapping. ${ }^{31}$

We propose here a method to trim the dispersion of strong index contrast waveguides using a self-adaptive boundary condition to significantly extend the bandwidth of Kerr comb spectra. This boundary condition was originally introduced in Ref. 32 for multimode optical waveguides in which an equivalent potential well ${ }^{33-35}$ in the waveguide transverse direction was achieved by the use of a lateral index profile, enabling an automatic phase-matching condition of degenerate fourwave-mixing processes. We explore here the properties of such waveguides, now operating in the single mode regime, and investigate their use for frequency comb generation. Our study shows that, in fact, such waveguides have an immense potential in terms of chromatic dispersion engineering, which can be made minimal or slightly abnormal in a wide spectral band, while being controllable by the geometrical parameters of the structures. The interest lies in the general nature of the proposed method, which can be implemented on various photonic platforms and can also be applied to waveguides with high core-cladding index contrasts. The metric used in this study is the spectral bandwidth of the Kerr frequency combs achieved from this approach and the comparison with standard silicon waveguides.

\section{Comb Bandwidth of Silicon Step-Index Waveguide}

The generation of Kerr frequency combs is traditionally achieved in a Kerr nonlinear ring/disk resonator, and the overall process of comb generation has been discussed in previous works. $^{12,36-51}$ A narrowband continuous optical source (laser) is centered close to an optical resonance of the ring resonator. If the chromatic dispersion of the ring is well chosen, it can result in a cascade of FWM processes in the frequency domain, which leads to the generation of a frequency comb. From a temporal point of view, the generation of such a broad-spectrum comb corresponds to a regime of short solitonic pulses or even, in the best of cases, to a regime of monosoliton propagation in the ring. The full dynamics of the comb generation process in which a Kerr microring resonator is fed by a side-coupled waveguide can be quantitatively described by solving the nonlinear Schrödinger equation, in the form of the Lugiato-Lefever equation (LLE) $)^{39-45,47,51}$

$$
\begin{aligned}
\tau_{R} \frac{\partial}{\partial t} A(t, \tau)= & \sqrt{\kappa} A_{\mathrm{in}}(\tau)-\left(\frac{\kappa}{2}+i \delta_{0}\right) A(t, \tau) \\
& +\left[i \sum_{k=2}^{n} \frac{\beta_{k}}{k !}\left(i \frac{\partial^{k}}{\partial \tau^{k}}\right)+i \gamma|A(t, \tau)|^{2}\right. \\
& -\frac{\beta_{2 \mathrm{PA}}}{2 A_{\mathrm{eff}}}|A(t, \tau)|^{2}-\frac{\beta_{3 \mathrm{PA}}}{3 A_{\mathrm{eff}}^{2}}|A(t, \tau)|^{4}-\frac{\alpha}{2} \\
& \left.-\frac{\alpha_{\mathrm{FCA}}}{2}\right] L A(t, \tau),
\end{aligned}
$$

where $A(t, \tau)$ is the pulse amplitude described by the time variable $\tau$ in a time frame corresponding to each round trip, $t$ stands for the accumulated calculation time while $\tau_{R}$ is the roundtrip span, $\beta_{k}$ is the $k$ 'th derivative of the wavevector with respect to the angular frequency $\beta_{k}=\frac{\partial^{k} \beta}{\partial \omega^{k}}$ (e.g., $\beta_{2}$ is the group velocity dispersion), $\gamma$ is the Kerr nonlinear parameter, $\alpha$ is the linear waveguide loss, $\beta_{2 \mathrm{PA}}$, and $\beta_{3 \mathrm{PA}}$ stand for the two-photon ${ }^{51-53}$ and three-photon absorption coefficients, ${ }^{54,55}$ respectively, and $A_{\text {eff }}$ is the waveguide effective mode area. The free-carrier effects $^{56,57}$ induced by multiphoton absorption are considered here through the $\alpha_{\mathrm{FCA}}$ coefficient, which is governed by the carrier density $N(t, \tau)$. The carrier density $N(t, \tau)$ is dynamically related to the intracavity power and the carrier lifetime $t_{\mathrm{FC}}$ through $\frac{\partial}{\partial \tau} N(t, \tau)=\frac{\beta_{2 \mathrm{PA}}}{2 \hbar \omega A_{\mathrm{eff}}^{2}}|A(t, \tau)|^{4}+\frac{\beta_{3 \mathrm{PA}}}{3 \hbar \omega A_{\mathrm{eff}}^{3}}|A(t, \tau)|^{6}-\frac{N(t, \tau)}{t_{\mathrm{FC}}}$. The pump is characterized by its amplitude $A_{\text {in }}$ and $\delta_{0}$, which describes the phase detuning between the pump frequency $\omega_{0}$ and the closest $n$ 'th-order cold-cavity resonant frequency $\omega_{n}$ of the ring resonator.

For the purpose of making reasonable comparison with previously proposed architectures and sticking to the fact that a PIN structure is sometimes needed to obviate free carrier accumulation issues, ${ }^{14,53} \mathrm{a}$ rib-shaped waveguide structure can be reasonably considered in a first approach, as shown in the inset of Fig. 1(a). The dispersion curve from Ref. 14 is reproduced in Fig. 1(a) (gray solid line), quantitatively through estimating $D=\frac{-2 \pi c}{\lambda^{2}} \cdot \frac{\mathrm{d}^{2} \beta}{\mathrm{d} \omega^{2}}$. Other compressed rib waveguides geometries (340-nm-thick silicon film, without SiN cladding) with rather similar dispersion properties but centered at smaller wavelengths are also chosen (orange and red curves), as shown in Fig. 1(a). Two configurations based on this thickness are shown in the inset, with only a difference in the waveguide width. Compared with a fully etched strip waveguide with the same or even a thinner thickness, these rib waveguides present a lower dispersion in the considered wavelength range. Four working points, including that of Ref. 14 (point 0), one for the 950nm rib waveguide width configuration (point 1), and two for the 1050-nm wide rib case (points 2 and 3), are selected for a further investigation of the dispersion-governed comb spectrum properties. 


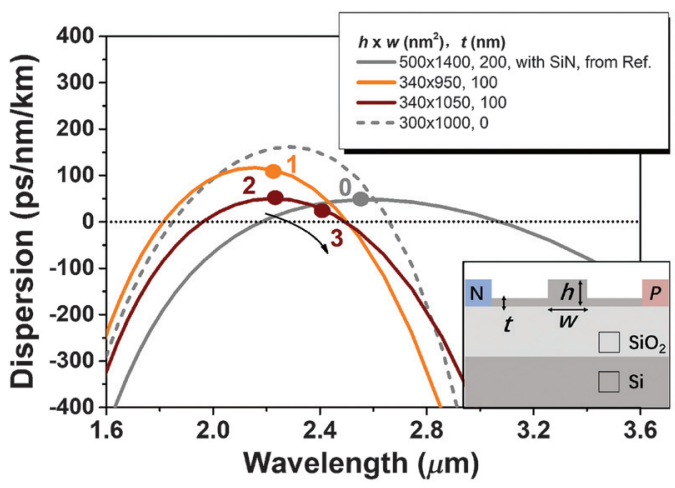

(a)

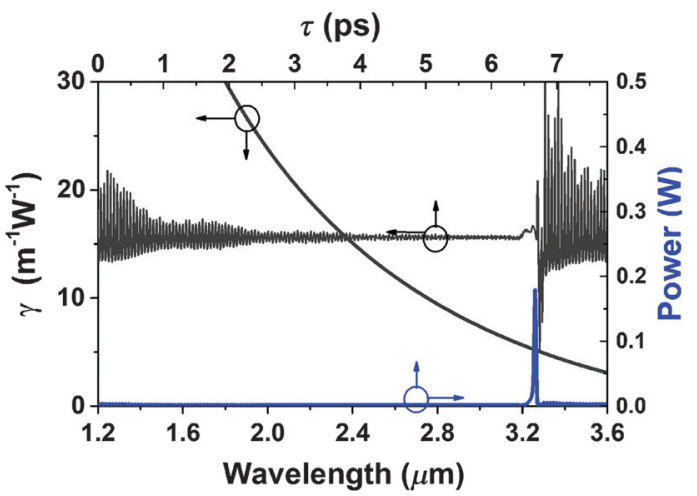

(c)

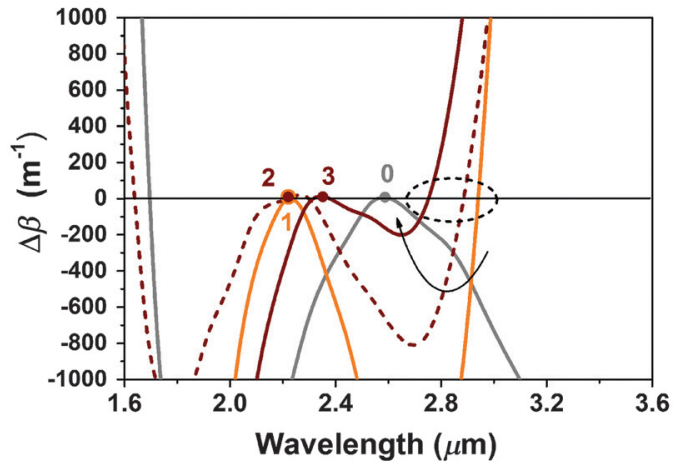

(b)

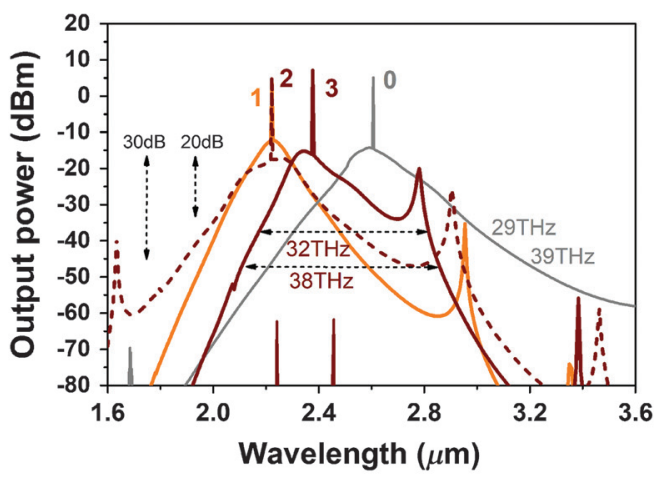

(d)

Fig. 1 (a) Dispersion curves of silicon-on-insulator (SOI) waveguides with different dimensions, including the one (gray line) from Ref. 14. Inset: Cross-section of a silicon ring resonator used for Kerr frequency comb modeling. (b), (d) The wavevector mismatch and associated spectra of frequency combs, with the working points indicated in (a). The near-bottom spectra are the corresponding primary comb lines. (c) The wavelength-dependent Kerr coefficient and its interpretation in the time domain in the single-soliton comb state, indicated by smooth and modulated curves in black. The pattern of the temporal soliton is also given in blue, corresponding to the upper $(\tau)$ and right (power) axis.

Due to the chromatic dispersion, light propagating along the ring resonator with different frequencies features different group velocities. The wavevector $\beta$ at the frequency $\omega$, will deviate from the wavevector of the pump $\left(\beta_{0}\right.$ at frequency $\left.\omega_{0}\right)$ by $\beta_{0}+\beta_{1}\left(\omega-\omega_{0}\right)$. Using Taylor's expansion, this mismatch is noted as $\Delta \beta=\left[\beta-\beta_{0}-\beta_{1}\left(\omega-\omega_{0}\right)\right]=\frac{\beta_{n}}{2}\left(\omega-\omega_{0}\right)^{2}+\ldots+$ $\frac{\beta_{n}}{n !}\left(\omega-\omega_{0}\right)^{n}$, which is considered an operator $i \sum_{k=2}^{n} \frac{\beta_{k}}{k !}\left(i \frac{\partial^{k}}{\partial \tau^{k}}\right)$ in Eq. (1). This classical figure of merit for evaluating a Kerr comb bandwidth ${ }^{13,15}$ is usually called "integrated dispersion" and is expressed in units of the microring free spectral range number. We prefer here to consider it as the dispersion "wavevector mismatch" consistent with its native expression. In Fig. 1(b), we show the wavevector mismatch $\Delta \beta$ for the different waveguide configurations mentioned above. Points 0,1 , and 2 are very typical operating points where $\Delta \beta$ rapidly evolves from $\Delta \beta=0$ with near-parabolic-shape local traces and increases back again with a second $\Delta \beta=0$ intersection where Cherenkov radiations are generated ${ }^{15}$ [see also the secondary spectral peaks in Fig. 1(d) for several waveguide configurations close to the secondary dispersion cancellation points]. Compared with point 1 , points 0 and 2 show smaller dispersion values (and possibly narrower anomalous regions) and therefore smaller deviations from the $\Delta \beta=0$ condition, which leads to larger spectrum bandwidth, as shown in Fig. 1(d). Parameters used here have been directly extracted from Ref. 14, i.e., the pump power is fixed to $150 \mathrm{~mW}$, a ring radius of $100 \mu \mathrm{m}$ is considered, and a free-carrier lifetime of $10 \mathrm{ps}$ is adopted, while propagation losses of $0.7 \mathrm{~dB} / \mathrm{cm}$ are assumed, and critical coupling conditions are retained. The wavelength-dependent Kerr nonlinear coefficient $^{4-6,21,28}$ was obtained using $\gamma=\frac{2 \pi n_{2} \eta}{\lambda A_{n \text { eff }}} \cdot\left(\frac{n_{g}}{n_{\mathrm{Si}}}\right)^{2}$, where $n_{2}, \eta$, and $A_{n \text { eff }}$ are the nonlinear index of silicon, the interaction overlap between the electric field and the silicon core, and the effective mode area, respectively. $n_{g}$ and $n_{\mathrm{Si}}$ are the group index of the optical mode and the material index, respectively. The interpretation of the nonlinear coefficient in the time domain of the single-soliton state, labeled here by a blue line, is also shown in Fig. 1(c) in black. We clearly see that even though the nonlinear coefficient decreases rapidly with increasing wavelength due to an enlarged mode area, the nonlinear coefficient is more or less anchored to the value obtained at the pump wavelength, being modulated only near the soliton position where energy from all frequencies comes to make a contribution.

One of the possible ways to expand the frequency comb spectrum is to shift the working point to a position closer to $\Delta \beta=0$ on the same curve. Comparing point 3 to point 2 , 
it can be observed that this action leads to the flattening of the wavevector mistmach $\Delta \beta$ [Fig. 1(b)] and therefore to a bandwidth improvement in Fig. 1(d). Configuration using working point 3 presents a comb bandwidth that is comparable (more precisely, a bit better) to that reported in Ref. 14 and is thus used hereafter as the reference case. The primary comb lines ${ }^{12,13,15}$ that are generated in the early stage of the comb generation process provide information that is useful for evaluating the bandwidth of parametric process; ${ }^{24-26}$ it is also plotted for point 3 at the bottom of Fig. 1(d). This is considered by the phase matching condition with the nonlinearity-induced phase and the group velocity dispersion taken into account and is approximately described using ${ }^{23,24}$

$\Delta \beta_{\text {total }}=2 \gamma P_{\text {intra }}-D \frac{\lambda^{2}}{2 \pi c} \cdot(\Delta \omega)^{2}$.

Here, $P_{\text {intra }}$ is the intracavity power, while $\Delta \omega$ is the frequency distance between the generated waves (idle/signal). These primary frequency lines are critical to the following subcombs and to the overall comb shape (and its bandwidth) around the pump line. It is thus very interesting to reduce and engineer the dispersion coefficient $D$ globally while keeping the other parameters fixed $\left(\gamma, P_{\text {in }}\right.$, etc.). ${ }^{22-26}$ This matter is discussed in the next section of the article.

\section{Silicon Frequency Comb with Engineered Dispersion in Self-Adaptive Boundary Waveguides}

In a standard step-index single-mode waveguide like the one described in Fig. 2(a), several wavelengths actually have the same physical boundaries. When the light wavelength increases, a more and more significant fraction of the mode energy is pushed out of the waveguide core into the cladding material. To illustrate this point, in Fig. 2(b), we consider the dispersion curve of a $750 \mathrm{~nm} \times 300 \mathrm{~nm}$ SOI waveguide (single-mode for $\lambda>2.1 \mu \mathrm{m}$ ). In view of this curve, it is quite remarkable that the

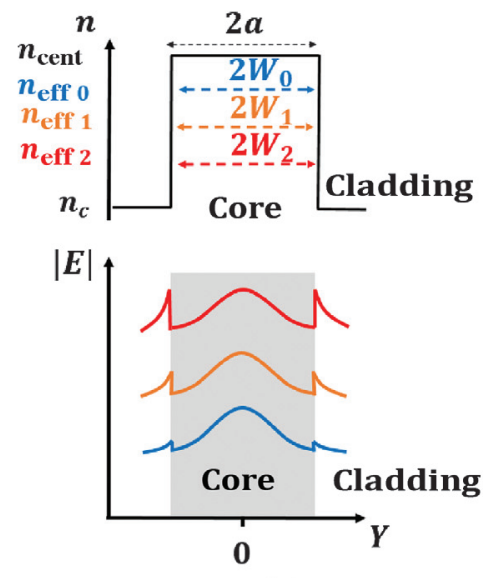

(a)

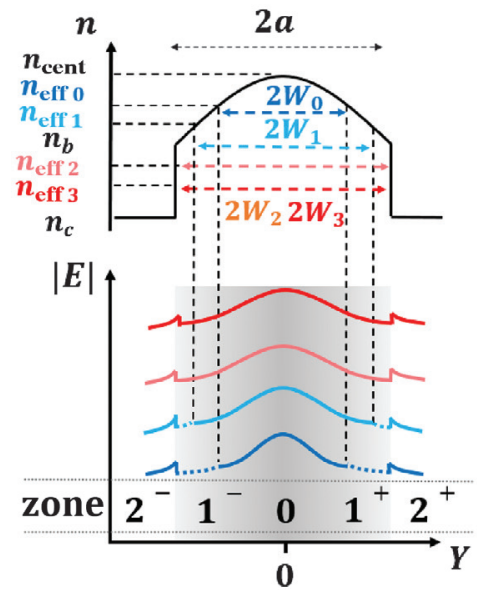

(c)

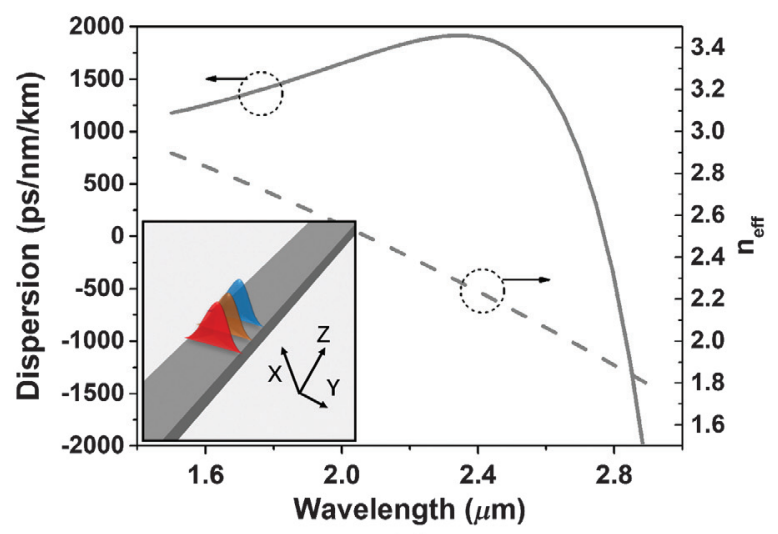

(b)

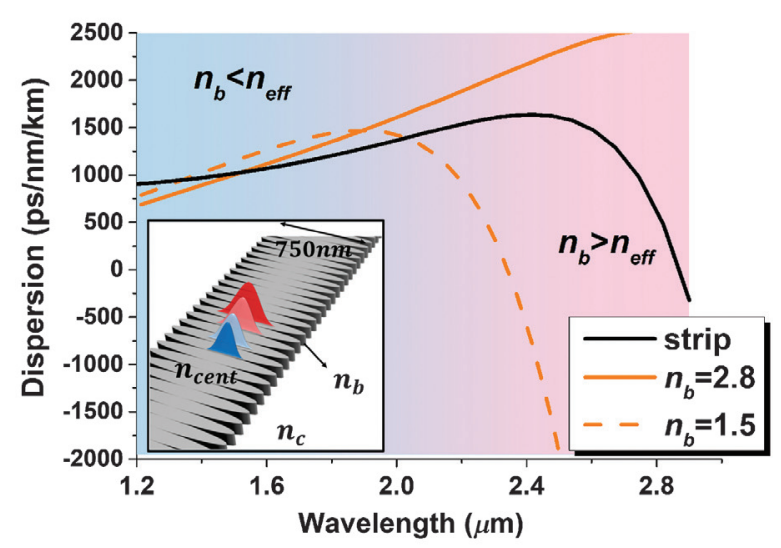

(d)

Fig. 2 (a) Schematics of a step-index waveguide. (b) The chromatic dispersion parameter $(D)$ of a silicon waveguide with a width and a height of 750 and $300 \mathrm{~nm}$, respectively $[n=3.48$ for the waveguide core, with an air upper cladding and silica $(n=1.44)$ for the bottom cladding]. (c) Schematics of a graded-index waveguide ensuring a self-adapting (SAB) condition (as defined in the text) and operating in a single-mode regime in the explored wavelength range. (d) Chromatic dispersion parameter $D$ of the waveguide mode. The width and height of the waveguide are 750 and $340 \mathrm{~nm}$, respectively. 
variation of the chromatic dispersion of the mode remains moderate up to about $2.7 \mu \mathrm{m}$. Then, its evolution becomes very fast. This illustrates the notable point that will guide our strategy to optimize the geometry of optical waveguides for the generation of Kerr frequency combs: the chromatic dispersion of the waveguides is actually strongly related to the degree of spatial confinement of the guided mode. In situations in which the mode cannot extend laterally due to spatial confinement from fixed boundary conditions, its chromatic dispersion amplitude tends to increase. One possible way to limit the waveguide mode dispersion is therefore to ensure a smooth spatial lateral confinement and to minimize the effect of the lateral waveguide boundaries by allowing the electromagnetic guided mode to spatially spread as freely as possible. Investigating this kind of waveguide modes has led us to identify a so-called self-adaptive boundary $(\mathrm{SAB})$ condition. ${ }^{32}$

With this objective in mind, we decided to explore the use of SAB waveguides ${ }^{32}$ within the perspective of using their unusual dispersion properties to generate Kerr frequency combs. Figure 2(c) shows a typical lateral index profile of an SAB waveguide, where the longitudinal axis of the upper diagram and the intensity of the gray region of the lower diagram both represent the material index of the waveguide core. A gradedindex profile along the transverse direction of the waveguide is used here, with $n_{\text {cent }}, n_{b}$, and $n_{c}$ representing the material index at waveguide center, at the waveguide inner edge, and the one of the cladding region. In a traditional strip waveguide (i.e., $n_{\text {cent }}=n_{b}$ ), the standing-wave region is confined by the boundary at the $n_{b} / n_{c}$ interface since $n_{b}>n_{\text {eff }}$. The standing-wave region is then the same for all wavelengths. In contrast, in a waveguide like the one represented in Fig. 2(c), if $n_{b}<n_{\mathrm{eff}}$, then the standing wave region is, instead, confined by the points where the material and the mode effective index values are equal. Since the mode effective index depends on the light wavelength, this leads to confining regions of different widths for different wavelengths, as illustrated in Fig. 2(c). In this situation, each confined field at a given wavelength automatically adapts its profile to a given spatial extension. To interrogate the effect of this $\mathrm{SAB}$ on the waveguide dispersion, the dispersion curves of a strip and two gradual SAB waveguides are put together for comparison in Fig. 2(d). Such graded-index profiles can be practically achieved by relying on the toolbox of subwavelength waveguide engineering, ${ }^{58-60}$ as we mentioned in Ref. 32. As shown in the inset of Fig. 2(d), the waveguide is composed by a periodical corrugation in which the individual period is itself characterized by a unitary silicon filling factor at the center and a reduced one at the edge. Using such a typical patterned structure, an equivalent material index can be flexibly designed. ${ }^{32}$ In the precise study case reported in Fig. 2(d), the period, thickness, and width of the stair-case graded index waveguide were chosen as 150,340 , and $750 \mathrm{~nm}$, respectively.

The dispersion of the strip waveguide displays a trend that is similar to the one of Fig. 2(b), highlighting a rapid change at long wavelengths. When the SAB condition is applied, the dispersion in Fig. 2(d) can actually be separated in two regions: the left part (blue region) corresponding to the $n_{b}<n_{\text {eff }}$ condition and the right one (red region) for $n_{b}>n_{\text {eff }}$. Dispersion of a strip waveguide and SAB waveguide with $n_{b}=1.5$ and $n_{b}=2.8$ is shown in Fig. 2(d) for comparison. The SAB allows us to trim the dispersion of the "long" wavelength where $n_{b}<$ $n_{\text {eff }}$ and the wave is confined by the index contrast of $n_{b} / n_{c}$ with the phase integral strongly dependent on the index $n_{b}$.
In contrast, since the short wavelength $\left(n_{b}>n_{\text {eff }}\right)$ is confined by the effective width of the waveguide where the electric field passes smoothly, the dispersion does not vary a lot. The selfadaptive response can be achieved in a continuously varying index profile, or in a discrete scheme, as shown in Fig. 3(a), where a multilevel silicon segment waveguide is described. This structure is, on the one hand, compatible with the liberation of the silicon waveguide from the silica substrate for eliminating the absorption of $\mathrm{SiO}_{2}$ for wavelengths higher than $3.6 \mu \mathrm{m}$; on the other hand, it provides room for the possible realization of a lateral PIN structure if needed for sweeping out free-carriers through a reverse applied bias, as shown in the inset.

To further expand the previous ideas, we now illustrate the proposed principle with a few typical examples chosen for their applicative relevance. The silicon thickness is chosen first at $340 \mathrm{~nm}$ as proposed in the reference structure in Fig. 1, which represents an appropriate value for optimizing the waveguide dispersion in the 2 to $4 \mu \mathrm{m}$ wavelength range. The length and width of the nanoarms are fixed at $L_{\text {arm }}=50 \mathrm{~nm}$ and $w_{\text {arm }}=$ $2.5 \mu \mathrm{m}$, which are values that are compatible with deep ultraviolet immersion lithography fabrication techniques. Let us first consider bilevel cases in which the value of $w_{2}$ in Fig. 3(a) is zero. Four configurations are plotted for comparison. The corresponding parameters are listed in the figure and figure caption. As the SAB condition can flatten the mode anomalous dispersion level for wavelengths not confined by the outer boundary, ripples containing two local peaks of anomalous dispersion can be expected using a bilevel SAB waveguide geometry, as observed in Fig. 3(b). The period of Bragg reflection is around $430 \mathrm{~nm}$, which is much larger than the longitudinal period that we consider $(240 \mathrm{~nm})$. Accordingly, this small period creates a bandgap only for wavelengths below $1.47 \mu \mathrm{m}$, which is far from our pump wavelength. This enables the periodic structure to operate as a homogeneous material ${ }^{58-60}$ in which the frequency-wavevector curve is much less dispersive than those near the band edge. Despite the fact that photonic bandgap effects from this periodic structure do not spoil the predicted dispersion and the overall validity of the proposed design, previous studies ${ }^{61,62}$ have indicated that splitting of some resonant peaks can be observed under a certain grating phase condition. However, this high-order grating effect is much slighter than the first one and is not included in our discussion. In these cases, a moderate index contrast with a filling factor varying from 1 to 0.66 (i.e., $L_{1} / p=1$ ) is chosen. As observed in Fig. 3(b), the whole wavelength range extending from 2.2 to $3.4 \mu \mathrm{m}$ is greatly flattened. Compared with those classical strip/ rib waveguides where the dispersion curve normally displays a parabolic shape, there is more than one local peak (e.g., one at $2.3 \mu \mathrm{m}$ and one at $3.3 \mu \mathrm{m}$ ) existing in an anomalous region, which gives us a new degree of freedom for dispersion engineering purpose. In particular, a small normal-dispersion region (gray zone) is squeezed out between these two wide anomalous regions (highlighted by blue and purple colors, from left to right). We also emphasize that the equivalent index of the outermost subwavelength cladding is $\sim 1.84$ compared with the core index $\sim 3.48$, which promises a high index contrast and tight mode confinement. The dispersion distortion associated with the bending radius could be seamlessly addressed by increasing the ring radius or by engineering the filling factor of the waveguide.

Two working points are selected for the calculation of the wavevector mismatch $\Delta \beta$, as shown in Fig. 3(c). The curve 


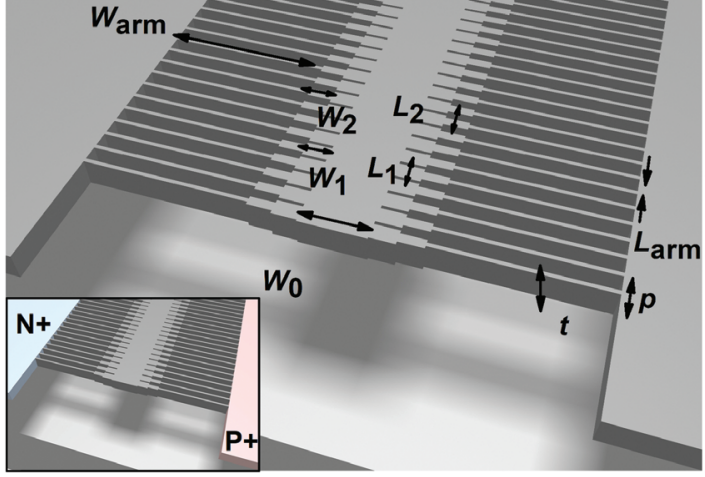

(a)

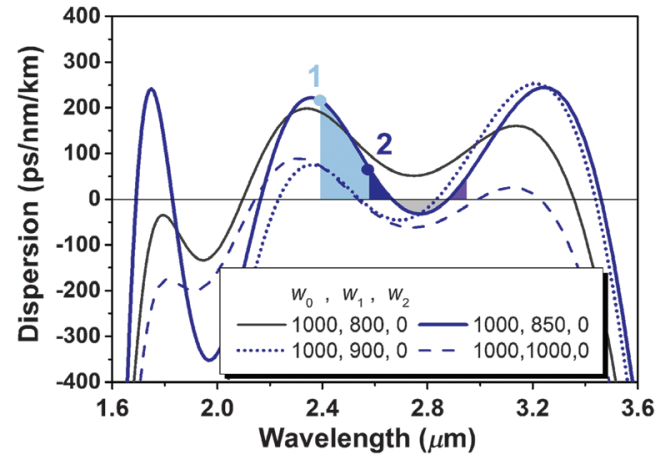

(b)

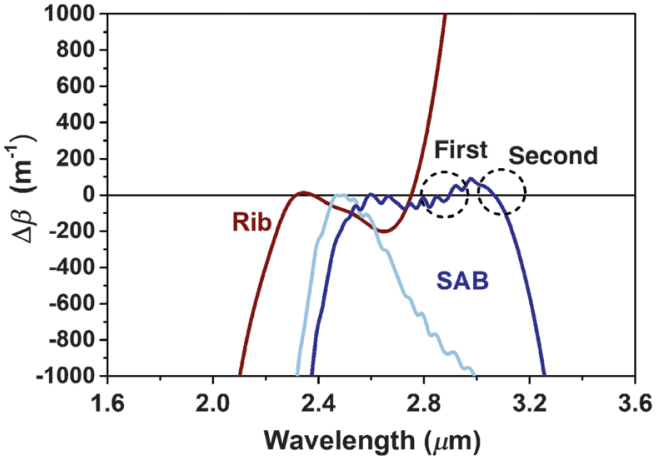

(c)

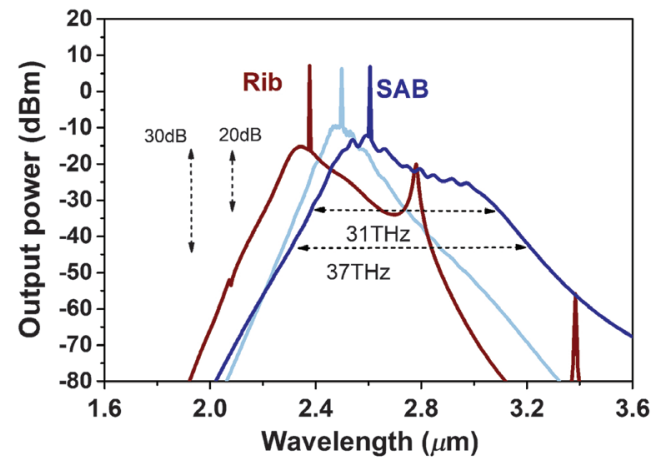

(d)

Fig. 3 (a) Schematic of a graded-index waveguide ensuring a self-adapting (SAB) condition. Inset: Possible a configuration comprising doped regions for free-carrier extraction. (b) Chromatic dispersion parameter $D$ of the four possible configurations of the proposed bilevel geometry. The silicon height is $t=340 \mathrm{~nm}$. (c) The calculated wavevector mismatch and (d) the single-soliton comb spectrum for our proposed SAB waveguide, at the working point in (b), compared with the reference rib microring described in Fig. 1 (red color). The two predicted Cherenkov radiation points are marked by the dashed circles in (c).

with point 1 shows a typical parabolic shape due to the high dispersion value and the rapid change of dispersion. However, by pushing it to point 2 , two intersection points (therefore two Cherenkov radiation regions) with $\Delta \beta=0$ are now achieved; they are highlighted by the black dashed circles. The consecutiveness of these two intersections, only spaced by $\sim 300 \mathrm{~nm}$, forces the whole curve to be flattened. Consequently, even better results than the reference one (red curve) are then obtained. Though fourth-order dispersion ${ }^{15,21}$ and even higher-order dispersion ${ }^{63}$ have been adopted for extending the zero-GVD working point, an improvement in comb bandwidth has not yet been witnessed. Flat dispersion has been achieved in a similar way in a vertical-slot waveguide, ${ }^{64}$ but the heterogeneous implementation was less attractive compared with our monolithic approach. The corresponding single-soliton comb spectra obtained by solving the LLE are given in Fig. 3(d). Since experimental demonstration ${ }^{65-68}$ already indicated loss levels smaller than $3 \mathrm{~dB} / \mathrm{cm}$ and according to previous experiments carried out on similar sub-wavelength waveguide structures, a linear waveguide loss level of $2 \mathrm{~dB} / \mathrm{cm}$ was considered here. The considered radius is the same as the one of the reference rib waveguide, i.e., $100 \mu \mathrm{m}$. To overcome the effect of the loss and enable the generation of a comb state, the pump power is slightly increased to $200 \mathrm{~mW}$. Note that critical coupling is always considered. The frequency detuning for achieving the final single-soliton comb is $1.513 \mathrm{GHz}$ from the initialization. In all of the cases considered here, the two-photon-induced and three-photon-induced absorption lead to extra loss of not more than 0.001 and $0.01 \mathrm{~dB} / \mathrm{cm}$, in which the additional loss penalty is much smaller than the linear loss level itself. The free carrier lifetime considered in this case is 50 ps. From our model, we estimated the influence of a free-carrier lifetime ranging from 10 to $100 \mathrm{ps}$ and observed, in the considered conditions, a weak influence of its value on the comb dynamics and the required pumping power. In a complementary fashion, we noticed that free-carriers exert a strong influence on the whole process only when their lifetime is larger than $200 \mathrm{ps,} \mathrm{in} \mathrm{which}$ case the carrier density can reach a few $10^{16} \mathrm{~cm}^{-3}$, which in return puts strong demand on the pump. Since the three-photon absorption process increases more quickly than the Kerr gain, at some point, comb generation is then totally prohibited for any pump power level. To sum up, we observed a lifetime cut-off with respect to each waveguide configuration. Regarding the main case corresponding to a propagation loss of $2 \mathrm{~dB} / \mathrm{cm}$, the cut-off lifetime was estimated to be around 220 ps. The situation could obviously be eased by lowering the linear loss. For instance, for propagation losses of $0.5 \mathrm{~dB} / \mathrm{cm}$, the cut-off lifetime increases to $420 \mathrm{ps}$.

As shown in Fig. 3(b), the accumulated wavevector mismatch from the deep-blue anomalous region is soon compensated for 
by the gray normal-dispersion region, which gives the first Cherenkov radiation. The remaining part of this gray region, however, is compensated for again by the right anomalous region (purple), which leads to the generation of the second Cherenkov radiation. These results evidence the potential of SAB waveguides for flattening the overall waveguide dispersion profiles and forcing the wavevector mismatch to intersect the $\Delta \beta=0$ axis twice without much deviation from it, which is difficult to achieve with traditional step-index waveguides of strip or rib shapes.

To improve the performance, a trilevel case $\left(w_{2} \neq 0\right)$ is considered to locally engineer the dispersion with an additional degree of freedom. As shown in Fig. 4(a), by varying the width of these three sections, we open a window of low-anomalous dispersion in a large wavelength range of up to $\sim 1000 \mathrm{~nm}$, while small areas of each zone are achieved. The filling factor of Secs. 1 and 2 are fixed at $L_{1} / p=0.8$ and $L_{2} / p=0.5$. The frequency detuning for achieving the final single-soliton comb is $1.272 \mathrm{GHz}$ from the initialization. Other parameters are the same as those previously mentioned, i.e., the loss, the carrier lifetime, the radius, and the pump power are $2 \mathrm{~dB} / \mathrm{cm}, 50 \mathrm{ps}$, $100 \mu \mathrm{m}$, and $200 \mathrm{~mW}$, respectively. As shown in Fig. 4(b), the wavevector mismatch $\Delta \beta$ is tremendously flattened near the axis from 2.4 to $3.2 \mu \mathrm{m}$ wavelengths, with again two intersection points and small ripples across and near the zero $\Delta \beta$ axis. This flattened dispersion profile assists us in achieving, as shown in Fig. 4(c), a comb spectrum that gives a 20/30-dB bandwidth of

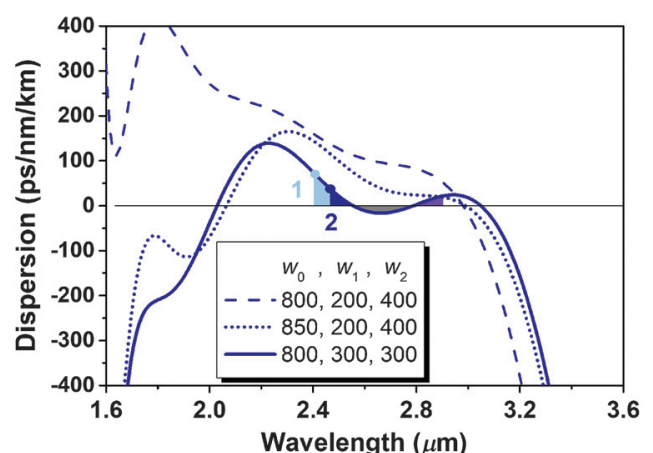

(a)

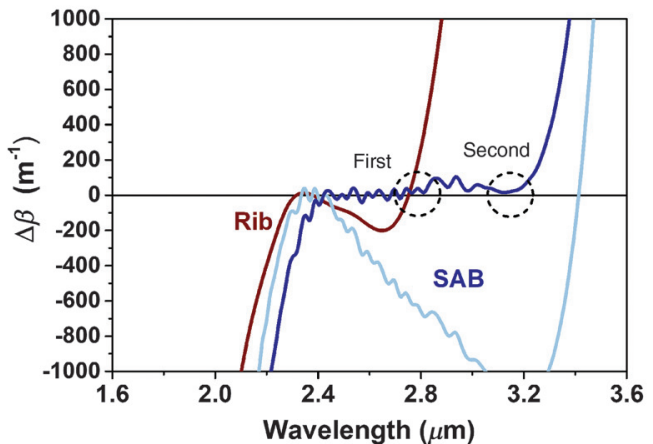

(b)

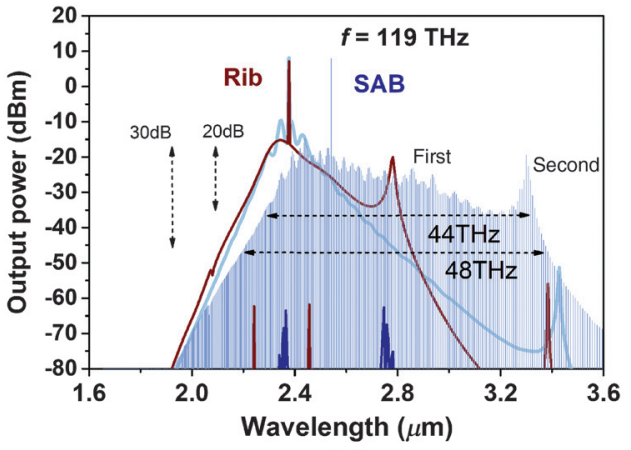

(c)

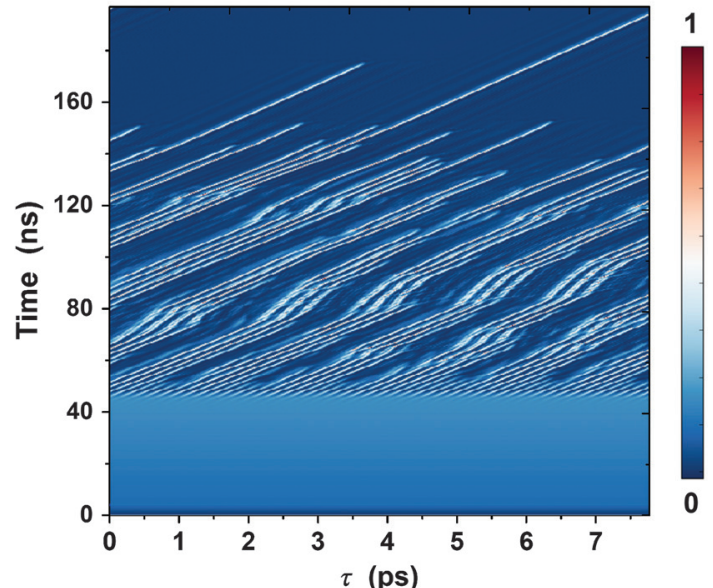

(d)

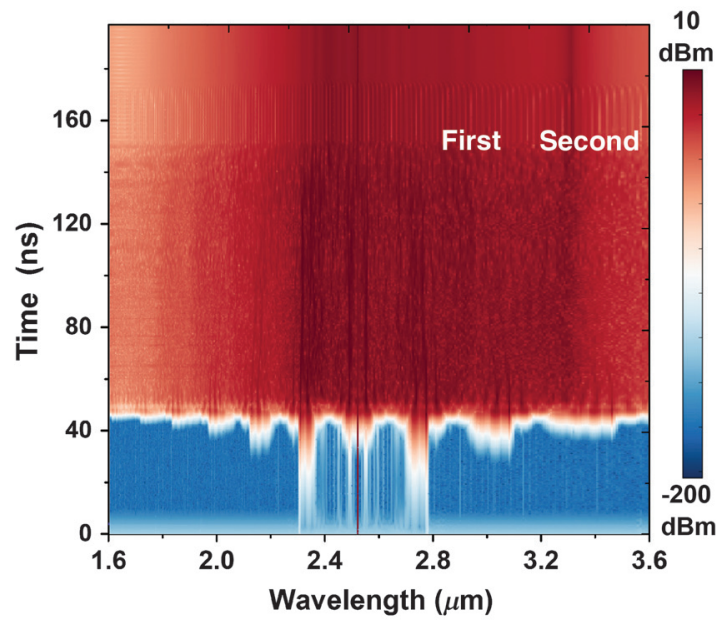

(e)

Fig. 4 (a) Chromatic dispersion parameter $D$ of four bilevel configurations with different parameters and based on the subwavelength silicon waveguides shown in Fig. 3(a). The silicon height is $t=340 \mathrm{~nm}$. (b) and (c) The calculated wavevector mismatch and single-soliton comb spectra corresponding to the working point in (a) and that from Fig. 1 (red color/reference Si rib waveguide). The near-bottom spectra are the corresponding primary comb lines. (d) and (e) The corresponding comb generation process of the comb in deep blue. The two predicted Cherenkov radiation points are marked by the dashed circles or the white labels. The frequency detuning for achieving the final single-soliton comb is $1.272 \mathrm{GHz}$ from the initialization. 
44/48 THz, which represents a great improvement compared with the optimal reference microring structure (red color). The two Cherenkov radiations can be distinguished from the smooth background. As visible, the primary comb lines of the SAB case (blue color), on the bottom part of Fig. 4(c), are much wider than that of the reference case. The evolution of the temporal and frequency patterns of the comb generation of the SAB waveguide comb with respect to point 2 is also presented in Figs. 4(d) and 4(e), respectively.

As already stated before, the loss, the carrier lifetime, and the pump power of the reference comb case were fixed at $0.7 \mathrm{~dB} / \mathrm{cm}, 10 \mathrm{ps}$, and $150 \mathrm{~mW}$, respectively, and while raising the loss and carrier lifetime to $2 \mathrm{~dB} / \mathrm{cm}$ and $50 \mathrm{ps}$ in our $\mathrm{SAB}$ case, respectively, the required pump power is only raised to $200 \mathrm{~mW}$. With this moderate pump power increase, the observed bandwidths have gained a large improvement, with a $20-\mathrm{dB}$ bandwidth raised from 32 to $44 \mathrm{THz}$ and a 30-dB bandwidth from 38 to $48 \mathrm{THz}$. Globally, these results prove that the nature of SAB waveguides implemented through a multisection lateral geometry opens up a new degree of freedom for engineering the needed waveguide dispersion and widening the Kerr frequency comb spectra.

\section{Conclusion}

We propose a general approach to trim and expand the spectral bandwidth of Kerr frequency combs in high-index-contrast optical platforms using self-adaptive boundary waveguides enabled by subwavelength index engineering. As a matter of illustration and a prime example, this approach enabled us to flatten the dispersion of silicon (on-insulator) waveguides and squeeze a small normal-dispersion region sandwiched between two anomalous zones. This improved dispersion shape enables us to achieve two Cherenkov radiation points and substantially broaden the bandwidth of comb spectra between 2.2 and $3.4 \mu \mathrm{m}$ wavelengths. This strategy addresses the dispersion issue in high-index-contrast platforms and can be used to further explore versatile nonlinear applications in which the manipulation of energy spacing and phase matching is of primary importance. We believe that this new approach will have broad applications in integrated nonlinear photonics for the realization of frequency combs, supercontinuum sources, and other nonlinear effects applicable to signal processing, metrology, and spectroscopy on-a-chip.

\section{Appendix}

The dispersion of periodically segmented silicon waveguides and their mode profiles are obtained from the band analysis of FDTD simulation (Lumerical Inc.), while the comb generation is modeled with a self-built Python code. The Lugiato-Lefever equation is solved by applying a split-step Fourier integration scheme to Eq. (1), and multistep calculation is considered in every round trip. The influence of waveguide dispersion on the light pulse is considered in the Fourier frequency domain while the loss and parametric amplification are accounted for in the time domain. The initial power level in the cavity is set at $-200 \mathrm{dBm}$. The pump wave is continuously injected into the input waveguide; it is first set at a wavelength slightly bluedetuned from a certain cold-cavity resonance and then slowly scanned across the resonance to longer wavelengths with increasing times. During this scanning process, the modulation instability, primary frequency lines, and cascaded four-wave- mixing processes are observed successively with the increase of the intracavity power. The overall time range is considered from nanoseconds to microseconds to confirm the stability of the generation of the Kerr frequency combs.

\section{Acknowledgments}

We appreciate the help from the French national research agency (BRIGHT ANR project). The authors declare no competing interests.

\section{References}

1. G. P. Agrawal, Nonlinear Fiber Optics, 5th ed., Academic Press, Boston, Massachusetts (1989).

2. J. Hansryd et al., "Fiber-based optical parametric amplifiers and their applications," IEEE J. Sel. Top. Quantum Electron. 8(3), 506-520 (2002).

3. J. Leuthold, C. Koos, and W. Freude, "Nonlinear silicon photonics," Nat. Photonics 4(8), 535-544 (2010).

4. M. C. Borghi et al., "Nonlinear silicon photonics," J. Opt. 19, 093002 (2017).

5. M. A. Foster et al., "Broad-band optical parametric gain on a silicon photonic chip," Nature 441, 960-963 (2006).

6. A. C. Turner-Foster et al., "Frequency conversion over two-thirds of an octave in silicon nanowaveguides." Opt. Express 18(3), 1904-1908 (2010).

7. X. Liu et al., "Mid-infrared optical parametric amplifier using silicon nanophotonic waveguides," Nat. Photonics 4(8), 557-560 (2010).

8. S. Zlatanovic et al., "Mid-infrared wavelength conversion in silicon waveguides using ultracompact telecom-band-derived pump source," Nat. Photonics 4(8), 561-564 (2010).

9. X. Liu et al., "Bridging the mid-infrared-to-telecom gap with silicon nanophotonic spectral translation," Nat. Photonics 6(10), 667-671 (2012).

10. P. A. Del'Haye et al., "Optical frequency comb generation from a monolithic microresonator," Nature 450, 1214-1217 (2007).

11. Y. Okawachi et al., "Octave-spanning frequency comb generation in a silicon nitride chip," Opt. Lett. 36(17), 3398-3400 (2011).

12. T. Herr et al., "Universal formation dynamics and noise of Kerr-frequency combs in microresonators," Nat. Photonics 6(7), 480-487 (2012).

13. T. Herr et al., "Temporal solitons in optical microresonators," Nat. Photonics 8(2), 145-152 (2014).

14. A. G. Griffith et al., "Silicon-chip mid-infrared frequency comb generation," Nat. Commun. 6, 6299 (2014).

15. V. Brasch et al., "Photonic chip-based optical frequency comb using soliton Cherenkov radiation," Science 351(6271) 357-360 (2016).

16. X. Xue et al., "Second-harmonic-assisted four-wave mixing in chip-based microresonator frequency comb generation," Light: Sci. Appl. 6, e16253 (2017).

17. H. Guo et al., "Mid-infrared frequency comb via coherent dispersive wave generation in silicon nitride nanophotonic waveguides," Nat. Photonics 12(6), 330-335 (2018).

18. N. Singh et al., "Octave-spanning coherent supercontinuum generation in silicon on insulator from $1.06 \mu \mathrm{m}$ to beyond $2.4 \mu \mathrm{m}$," Light: Sci. Appl. 7, 17131 (2018).

19. B. Stern et al., "Battery-operated integrated frequency comb generator," Nature 562, 401-405 (2018).

20. T. J. Kippenberg et al., "Dissipative Kerr solitons in optical microresonators," Science 361(6402), eaan8083 (2018).

21. M. A. Foster et al., "Broad-band continuous-wave parametric wavelength conversion in silicon nanowaveguides," Opt. Express 15(20), 12949-12958 (2007). 
22. A. B. Matsko et al., "Clustered frequency comb," Opt. Lett. 41(21), 5102-5105 (2016).

23. M. Haelterman, S. Trillo, and S. Wabnitz, "Additive-modulationinstability ring laser in the normal dispersion regime of a fiber," Opt. Lett. 17(10), 745-747 (1992).

24. N. L. B. Sayson et al., "Octave-spanning tunable parametric oscillation in crystalline Kerr microresonators," Nat. Photonics 13(10), 701-706 (2019).

25. S. Fujii et al., "Octave-wide phase-matched four-wave mixing in dispersion-engineered crystalline microresonators," Opt. Lett. 44(12), 3146-3149 (2019).

26. J. Yang et al., "Coherent satellites in multispectral regenerative frequency microcombs," Commun. Phys. 3, 27 (2020).

27. S.-W. Huang, et al., "Mode-locked ultrashort pulse generation from on-chip normal dispersion microresonators," Phys. Rev. Lett 114, 053901 (2015)

28. B. Yao et al., "Gate-tunable frequency combs in graphene-nitride microresonators," Nature 558, 410-414 (2018).

29. A. Pasquazi et al., "Micro-combs: a novel generation of optical sources," Phys. Rep. 729, 1-81 (2018).

30. N. Picqué et al., "Frequency comb spectroscopy." Nat. Photonics 13, 146-157 (2019).

31. P. Feng, et al., "Dual-comb spectrally encoded confocal microscopy by electro-optic modulators," Opt. Lett. 44(11), 2919-2922 (2019).

32. J. Zhang et al., "Self-adaptive waveguide boundary for inter-mode four-wave mixing," IEEE J. Sel. Top. Quantum Electron. 26(2), 5100108 (2020).

33. M. Eichenfield et al., "Optomechanical crystals," Nature 462, 78-82 (2009).

34. F. Alpeggiani et al., "Effective bichromatic potential for ultra-high Q-factor photonic crystal slab cavities," Appl. Phys. Lett. 107(26), 261110 (2015).

35. A. Simbula, et al., "Realization of high-Q/V photonic crystal cavities defined by an effective Aubry-André-Harper bichromatic potential," Appl. Phys. Lett. 2(5), 056102 (2017).

36. J. M. Dudley et al., "Supercontinuum generation in photonic crystal fiber," Rev. Mod. Phys. 78, 1135 (2006).

37. V. E. Zakharov et al., "Exact theory of two-dimensional selffocusing and one-dimensional self-modulation of waves in nonlinear media," Sov. Phys. JETP 34, 62-69 (1972).

38. I. V. Barashenkov et al., "Existence and stability chart for the ac-driven, damped nonlinear Schrödinger solitons," Phys. Rev. E 54(5), 5707-5725 (1996).

39. K. Ikeda et al., "Multiple-valued stationary state and its instability of the transmitted light by a ring cavity system," Opt. Commun. 30(2), 257-261 (1979).

40. L. Lugiato et al., "Spatial dissipative structures in passive optical systems," Phys. Rev. Lett. 58(21), 2209-2211 (1987).

41. L. Lugiato et al., "From the Lugiato-Lefever equation to microresonator based soliton Kerr frequency combs," Trans. R. Soc. A 376(2135), 20180113 (2018).

42. M. Haelterman et al., "Dissipative modulation instability in a nonlinear dispersive ring cavity," Opt. Commun. 91(5), 401-407 (1992).

43. A. B. Matsko et al., "Mode-locked Kerr frequency combs," Opt. Commun. 36(15), 2845-2847 (2011).

44. S. Coen et al., "Universal scaling laws of Kerr frequency combs," Opt. Lett. 38(11), 1790-1792 (2013).

45. S. Coen et al., "Modeling of octave-spanning Kerr frequency combs using a generalized mean-field Lugiato-Lefever model," Opt. Lett. 38(1), 37-39 (2013).

46. T. Hansson et al., "Dynamics of microresonator frequency comb generation: models and stability," Nanophotonics 5(2), 231-243 (2016).

47. M. R. E. Lamont et al., "Route to stabilized ultrabroadband microresonator-based frequency combs," Opt. Express 38(18), 34783481 (2013).
48. H. Guo et al., "Universal dynamics and deterministic switching of dissipative Kerr solitons in optical microresonators," Nat. Phys. 13(1), 94-102 (2017).

49. Y. K. Chembo et al., "Spectrum and dynamics of optical frequency combs generated with monolithic whispering gallery mode resonators," Phys. Rev. Lett. 104(10), 103902 (2010).

50. Y. K. Chembo et al., "Modal expansion approach to opticalfrequency-comb generation with monolithic whispering-gallerymode resonators," Phys. Rev. A 82(3), 033801 (2010).

51. R. K. W. Lau et al., "Effects of multiphoton absorption on parametric comb generation in silicon microresonators," Opt. Lett. 40(12), 2778-2781 (2015).

52. A. D. Bristow et al., "Two-photon absorption and Kerr coefficients of silicon for 850-2200 nm," Appl. Phys. Lett. 90(19), 191104 (2007).

53. M. Först, et al., "High-speed all-optical switching in ion-implanted silicon-on-insulator microring resonators," Opt. Lett. 32(14), 2046-2048 (2015).

54. S. Pearl, et al., "Three photon absorption in silicon for 2300 3300 nm," Appl. Phys. Lett. 93(13), 131102 (2008).

55. F. Gholami et al., "Third-order nonlinearity in silicon beyond 2350 nm," Appl. Phys. Lett. 99(8), 081102 (2011).

56. D. K. Schroder et al., "Free carrier absorption in silicon," IEEE J. Solid-State Circuits 13(1), 180-187 (1978).

57. R. R. Vardanyana et al., "Modeling free carrier absorption in silicon," J. Contemp. Phys. 47(2), 73-79 (2012).

58. D. Ortega et al., "Analysis of "quasi-modes" in periodic segmented waveguides," J. Lightwave Technol. 17(2), 369-375 (1999).

59. R. Halir et al., "Waveguide sub-wavelength structures: a review of principles and applications," Laser Photonics Rev. 9(1), 25-49 (2015).

60. P. Cheben et al., "Subwavelength integrated photonics," Nature 560, 565-572 (2018).

61. X. Lu et al., "Selective engineering of cavity resonance for frequency matching in optical parametric processes," Appl. Phys. Lett. 105(15), 151104 (2014).

62. K. McGarvey-Lechable and P. Bianucci, "Bloch-Floquet waves in optical ring resonators," Phys. Rev. B. 97(21), 214204 (2018).

63. G. Moille et al., "Phased-locked two-color single soliton microcombs in dispersion-engineered $\mathrm{Si}_{3} \mathrm{~N}_{4}$ resonators," Opt. Lett. 43(12), 2772-2775 (2018).

64. Y. Guo et al., "Ultra-flat dispersion in an integrated waveguide with five and six zero-dispersion wavelengths for mid-infrared photonics," Photonics Res. 7(11), 1279-1286 (2019).

65. J. S. Penadés et al., "Suspended SOI waveguide with subwavelength grating cladding for mid-infrared," Opt. Lett. 39(19), 5661-5664 (2014).

66. J. S. Penadés et al., "Suspended silicon mid-infrared waveguide devices with subwavelength grating metamaterial cladding," Opt. Express 24(20), 22908-22918 (2016).

67. H. Lin et al., "Mid-infrared integrated photonics on silicon: a perspective," Nanophotonics 7(2), 393-420 (2018).

68. S. A. Miller et al., "Low-loss silicon platform for broadband mid-infrared photonics," Optica 4(7), 707-712 (2017).

Jianhao Zhang is currently a post-doctoral researcher with the Centre for Nanoscience and Nanotechnology (C2N) at Paris-Saclay University/ CNRS, France. He received his PhD from Paris-Sud University and Zhejiang University in 2019. His research interests include nonlinear optics, cavity opto-mechanics, integrated lasers, as well as interdisciplinary research merging optics and other research fields, within the scope of silicon photonics.

Vincent Pelgrin received his master's degree in fundamental physics and application majoring photonics from Rennes 1 University in France in 2019. He is currently a PhD student in the Center for Nanoscience and Nanotechnology (C2N) at Paris Saclay University and in the Department of Electronics and Nanoengineering at Aalto University, Finland. 
His research topic mainly focuses on hybrid integrated silicon photonics using 2D materials to enhance nonlinear light-matter interaction.

Carlos Alonso-Ramos received his $\mathrm{PhD}$ in 2014 from the Universidad de Málaga, Spain, on the development of high-performance integrated photonic circuits for chip interconnects and next generation coherent transceivers. He is currently a CNRS Researcher with the Center for Nanoscience and Nanotechnology (C2N) in Palaiseau, France. His research interest includes the development of Si photonics devices and circuits in the near- and mid-infrared for applications in telecom, sensing, and quantum.
Eric Cassan was awarded a PhD in 2000, was successively associate professor and then full professor at Paris-Sud University. Since 2016, he has carried out his research themes within the Centre for Nanosciences and Nanotechnologies at Université Paris-Saclay/CNRS. The latest orientations of his work concern the realization of integrated functions exploiting nonlinear optical effects for the realization of light sources. $\mathrm{He}$ is the author of about 270 articles and invited oral communications at international conferences.

Biographies of the other authors are not available. 